\title{
On the Theoretical Analysis of Memristor based True Random Number Generator
}

\author{
Mesbah Uddin \\ The University of Tennessee \\ Knoxville, Tennessee \\ muddin6@vols.utk.edu
}

\author{
Md Sakib Hasan \\ The University of Tennessee \\ Knoxville, Tennessee \\ mhasan4@vols.utk.edu
}

\author{
Dr. Garrett S. Rose \\ The University of Tennessee \\ Knoxville, Tennessee \\ garose@utk.edu
}

\begin{abstract}
Emerging nano-devices like memristors display stochastic switching behavior which poses a big uncertainty in their implementation as the next-generation CMOS alternative. However, this stochasticity provides an opportunity to design circuits for hardware security. There are several examples in literature where the stochastic switching time of memristors are used as the source of entropy to build true random number generators (TRNGs). Software-based pseudorandom numbers may not be random enough for many different applications where true random numbers are a necessity. In this work, we have analyzed traditional TRNG designs that utilize memristors' switching time and evaluated them in varying operating conditions and with process variation in mind. Specifically, we have mathematically formulated how large process variation and strong temperature and voltage dependence of memristors can degrade the performance of these TRNGs. Depending on these analyses, we also have proposed a new way of designing memristive TRNG based on difference between stochastic high resistance states of a pair of memristors. Using simple probabilistic mathematics, we have evaluated our proposed method with existing ones and shown that our proposed design is robust in unfavorable environmental conditions and in the presence of large process variation where traditional TRNG bit quality degrades rapidly.
\end{abstract}

\section{CCS CONCEPTS}

-Security and privacy $\rightarrow$ Embedded systems security; Hardwarebased security protocols.

\section{KEYWORDS}

True random number generator; TRNG; RNG; memristor; Hardware security; memristor based RNG

\section{ACM Reference Format:}

Mesbah Uddin, Md Sakib Hasan, and Dr. Garrett S. Rose. 2019. On the Theoretical Analysis of Memristor based True Random Number Generator. In Great Lakes Symposium on VLSI 2019 (GLSVLSI'19), May 9-11, 2019, Tysons Corner, VA, USA. ACM, New York, NY, USA, 6 pages. https://doi.org/10.1145/ 3299874.3317981

Permission to make digital or hard copies of all or part of this work for personal or classroom use is granted without fee provided that copies are not made or distributed for profit or commercial advantage and that copies bear this notice and the full citation on the first page. Copyrights for components of this work owned by others than ACM must be honored. Abstracting with credit is permitted. To copy otherwise, or republish, to post on servers or to redistribute to lists, requires prior specific permission and/or a fee. Request permissions from permissions@acm.org.

GLSVLSI '19, May 9-11, 2019, Tysons Corner, VA, USA

(c) 2019 Association for Computing Machinery.

ACM ISBN 978-1-4503-6252-8/19/05 . .\$15.00

https://doi.org/10.1145/3299874.3317981

\section{INTRODUCTION}

With the emergence of billions of smart interconnected devices (Internet of things or IoT), hardware security is becoming more important that ever because of its ability to provide very lightweight and unique hardware specific secucirty solutions. Most, if not all security implementations require secret keys. Key generation software usually follows some complex algorithms to generate their secret keys randomly. However, these software-generated keys are at best pseudo-random in nature and may not fulfill randomness requirements of many applications. Therefore, there is a growing need for true random number generators (TRNGs) that can produce a stream of true random bits from hardware for cryptographic applications [5]. Using some stochastic and random physical phenomena within a circuit, these TRNGs produce a stream of random bits. True random numbers (TRN) are different from pseudo random numbers (PRN) where a seed is usually needed and the same stream of bits are produced if the initializing seed remains the same. A TRNG should ideally be insensitive to environmental variation, i.e. the source of entropy that produces the random bits should not be a strong function of temperature or supply voltage. Process variation should also have a small impact so that it doesn't disturb the randomness of the TRNG circuit. Naturally occurring random circuit phenomena are used to build TRNGs. These phenomena include clock jitter, random telegraph noise (RTN), thermal noise, metastable state, any quantum phenomena, etc.

Recently, stochasticity in novel nano-devices like memristors [11] is being explored to build TRNGs $[4,16]$. Memristors display probabilistic switching behavior, i.e. their switching time or memristive states can have different values at different periods of time [9]. Researchers have tried to use this as a source of randomness to build different hardware security primitives specifically physical unclonable functions (PUF) $[12,14]$, sneak-path based tag generation [7], random number generator [16] etc. In [16], probability distribution of memristors's OFF and ON states based on the switching time is used to design the MTRNG (memristor-based TRNG). In [6], researchers have utilized a differential readout scheme to harvest the RTN (random telegraph noise) in memristors along with LFSR (linear feedback shift register) to implement a TRNG. The differential nature of this circuit improves performance against varying temperature and power supply noise. Switching variability in memristor's set process is used to extract random numbers in [1]. In [4], authors have proposed TRNG design where the random switching time of a diffusive memristor is used as the source of entropy and is shown to produce true random numbers without any post-processing. This diffusive memristor is different than common metal-oxide memristors, does not require forming and reverts 
to its high resistance state spontaneously. However, this self-OFF switching $(\approx 1 \mathrm{~ms})$ property makes these memristors very slow.

The main purpose of this work is to analyze the underlying mathematical basis for traditional memristor based TRNGs. We have analyzed one such design and formulated how temperature, supply voltage change and process variation could affect the TRNG performance. To improve robustness against process variation and environmental changes, we have suggested a TRNG design that leverages the variability of high resistance state (HRS) of a memristor. This design uses relative measurements, i.e. the HRS of one memristor is compared with another to generate a random bit. Since HRS usually has shown higher variability compared to other memristor parameters [10], we have chosen HRS as the source of randomness for our design. The differential nature of this design makes it robust against process and environmental variation.

The contributions of this paper include

- Formulation of conventional memristor based TRNG design,

- Mathematical formulation of changes in performance of conventional design with outside effects,

- Proposition of a new twin memristor based TRNG design,

- Evaluation of both existing and proposed design against environmental changes,

- Evaluation of both design against process variation.

The paper is organized as follows: section 2 first presents a short introduction to memristor and its stochastic behavior. Section 3 derives the relationship of existing TRNG design with temperature shift and supply voltage and process variation. Section 4 presents and discusses our proposed design and again derives the relationship of this design with temperature, supply voltage and process variation. Section 5 presents the simulation results and explains the difference between these two designs. Finally, future prospects and concluding remarks are provided in section 6 and 7, respectively.

\section{BACKGROUND}

\subsection{Introduction to Memristor}

The memristor or ReRAM/RRAM (resistive random access memory) is one of the most promising post Moore-era devices because of their non-volatility, CMOS compatibility, low energy operation among other features [11]. Metal oxide memristors (metal-insulatormetal or MIM layer) are more common among various types of memristors. They are usually bi-polar, i.e. they have two stable resistive states namely high resistance state (HRS) or the OFF state and the low resistance state (LRS) or the ON state. Applying a minimum threshold voltage for a minimum amount of time switches a memristor from LRS to HRS or from HRS to LRS depending on the direction of applied voltage. The minimum threshold voltage and minimum time to switch from HRS to LRS are called positive threshold voltage and positive switching time, respectively, where from LRS to HRS, they would be called negative threshold and negative switching time, respectively. The transition from LRS to HRS is known as RESET where the transition from HRS to LRS is called SET. As memristors display RTN (random telegraph noise) i.e. their final HRS and LRS values along with threshold voltages and switching times are stochastic over time. Also, due to their nanoscale size and relatively immature fabrication process, memristors display a large die-to-die process variation $[10,15]$.

\subsection{Stochasticity in Memristor}

As mentioned before, in metal-oxide memristors, threshold behavior is observed. This means when the applied voltage is larger than a threshold voltage for at least the minimum switching time, the memristor switches its state from one memristive (or resistive) state to another. Because of large manufacturing process variation, memristors display a wide range of characteristics. Also, because of their underlying physical nature of switching mechanism, they also display varied characteristics in time. Thus their switching process is stochastic i.e. the value of their memristive states, switching time and threshold voltages can display variation across time. Over a larger number of clock cycles, their stochastic behavior can be approximated as Gaussian or Normal distribution [17]. A memristive TRNG would try to extract the uncertainty in their switching over different cycles to generate stream of random bits.

\section{EFFECT OF ENVIRONMENTAL AND PROCESS VARIATION ON CONVENTIONAL DESIGN}

As mentioned in earlier sections, most of the existing memristor based TRNG designs take advantage of the stochastic switching time. Memristors display cycle-to-cycle variation in their characteristics. The switching time (positive or negative) of a memristor can be approximated to have a normal distribution over many cycles. This is formulated as:

$$
\mathbf{t}_{\text {swn }}=N\left(\mu_{t s w n}, \sigma_{t s w n}\right)
$$

and,

$$
\mathbf{P}\left(t \leq \mu_{t s w n}\right)=\mathbf{P}\left(t>\mu_{t s w n}\right)=0.5
$$

To reiterate, equation 1 describes that the switching time of a memristor is a Gaussian (Normal) random variable with mean $\mu_{t s w n}$ and standard deviation $\sigma_{t s w n}$. Therefore, equation 2 expresses the probability that a random switching time, $\mathrm{t} \epsilon \mathbf{t}_{\text {tswn }}$ to be less than or equal to the mean which is 0.5 or $50 \%$ according to the property of a Gaussian distribution. Thus if we determine the mean of that switching time $\left(\mu_{t s w n}\right)$ and apply a voltage greater than its threshold voltage to a memristor repeatedly for many cycles, then half of the time, memristor would switch to its other state and the other half of the time, it would not. This is the basis for most memristor based RNG where memristors's stochastic switching time is the source of entropy.

This method requires a precise pulse with pulse width being approximately equal to the mean switching time. Any clock jitter/skew or other source of noise could change this pulse width which may drastically change the overall switching probability. The switching time also depends heavily on the applied voltage magnitude across the memristor. Thus, any power supply noise would also strongly affect the switching time as well. Again, since the memristor's threshold voltages and memristive states are functions of temperature, temperature change would also affect the switching time and thus the overall switching probability. Thus any change in any of these factors would bring a change in the probability of switching and thus equation 2 would not hold for the changed distribution. Then the produced bit stream from that memristor could be heavily biased towards either a ' 1 ' or a ' 0 '. In the next few 
subsections, we are going to formulate the change in switching time distribution for these factors.

\subsection{Supply voltage variation}

To simplify our analysis and to get an approximate idea how voltage variation can affect the random number generator performance, we have considered a linear model for a memristor which was developed first by Mcdonald et.al. [8] and later improved in [12, 13] The RESET of a memristor (i.e. from LRS to HRS) is governed by this equation [13]:

$$
M\left(t_{i+1}\right)=M\left(t_{i}\right)+\frac{R_{d i f f} \Delta t V\left(t_{i+1}\right)}{t_{s w n} V_{t n}},
$$

where, $t_{s w n}$ and $V_{t n}$ are negative switching time and negative threshold voltage of a memristor, respectively. $R_{\text {diff }}$ is the difference between HRS and LRS. $M(t)$ presents the memristance and $V(t)$ represents the applied voltage across the memristor at a time instant, t. Equation 3 can be written as:

$$
\Delta M=\frac{R_{\text {diff }}\left|V_{\text {applied }}\right|}{t_{s w n} V_{t n}} * \Delta t,
$$

By integrating this equation 4 , we can establish a relationship between switching time and applied voltage. Let's assume that the applied voltage remains the same for one switching cycle, from equation 4 , we can estimate the actual switching time for any applied voltage:

$$
\begin{gathered}
\int_{L R S}^{H R S} d M=\frac{R_{\text {diff }}\left|V_{\text {applied }}\right|}{t_{s w n} V_{t n}} * \int_{0}^{t_{\text {sw,actual }}} d t \\
\Longrightarrow t_{s w, \text { actual }}=t_{s w n} * \frac{V_{t n}}{\left|V_{\text {applied }}\right|}
\end{gathered}
$$

The resulting relationship between applied voltage and actual time to switch is found to be proportional which is expected from a linear switching model of a memristor. If the change in applied voltage is limited ( $\max \pm 10 \%$ here), then a linear equation should provide reasonable accuracy. If $V_{\text {applied }}$ equals to the threshold $V_{t n}$, then the actual switching time is equal to the minimum switching time. However, from equation 6, a $\pm 10 \%$ change in $V_{\text {applied }}$ from $V_{t n}$ would result in a $(\approx-9 \%,+11 \%)$ change in actual switching time.

\subsection{Temperature variation}

Temperature directly affects memristive states and threshold voltages and thus in turn affect the actual switching time. From [2, 3], we can see threshold voltage magnitude decreases with increasing temperature within the range $\approx[0,100]^{0} \mathrm{C}$ and we can approximate the change with a linear equation as:

$$
V_{t n}(\theta)=V_{t n 0}\left[1+\alpha_{V t n}\left(\theta-\theta_{0}\right)\right]
$$

where, $V_{t n 0}$ is the threshold voltage at room temperature, $\theta_{0}=25^{0} \mathrm{C}$. From [3], the temperature coefficient for negative threshold voltage is calculated as $\approx-0.007 /{ }^{0} \mathrm{C}$. Therefore, for a $\Delta \theta$ temperature change, the new $\mathrm{V}_{t n}$ would be:

$$
V_{t n}(\theta)=[1-0.007 * \Delta \theta] * V_{t n 0},
$$
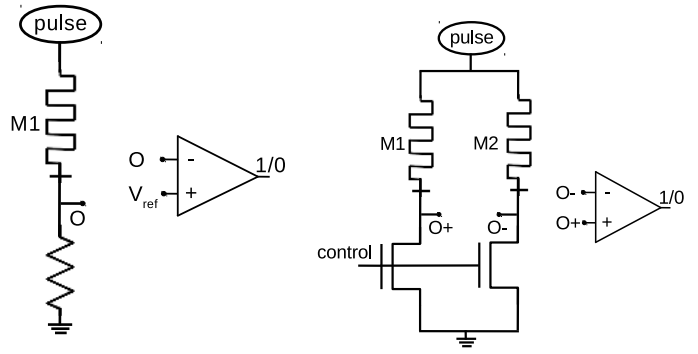

Figure 1: Conceptual circuit diagram of (left) conventional switching time based single memristor TRNG, and (right) the proposed twin memristor TRNG

The modified switching time would then be:

$$
t_{s w, \text { actual }}=t_{s w n} * \frac{[1-0.007 * \Delta \theta] * V_{t n 0}}{V_{\text {applied }}}
$$

\subsection{Process variation}

Because of large process variation of an emerging devices like memristor, all of its major parameters display a relatively larger variance. For existing RNG designs which are based on switching time, we are only interested in the variation of switching time. For this purpose, we have considered a $5 \%$ standard deviation $\left(\sigma_{t s w n}\right)$ on negative switching time, $\mathrm{t}_{\text {swn }}$.

$$
\mathbf{t}_{\mathrm{swn}}=N\left(\mu_{t s w n}, \sigma_{t s w n}\right), \text { die }- \text { to }- \text { die }
$$

where $\sigma_{t s w n}=0.05^{*} \mu_{t s w n}$. Thus for a $1 \sigma$ variation of this switching time, the shifted mean of this distribution would be:

$$
t_{s w, \text { actual }}=\left\{\begin{array}{ll}
1.05 * \mu_{t s w n}, & 1 \sigma \text { above mean } \\
0.95 * \mu_{t s w n}, & 1 \sigma \text { below mean }
\end{array} .\right.
$$

\section{PROPOSED TRNG DESIGN}

We have seen that process variation, temperature change and supply voltage variation can affect and thus shift the switching time distribution of a memristor. Therefore, any TRNG design based on the initial distribution of cycle-to-cycle switching time around the mean of this distribution would create biased proportion of ones and zeros and thus degrade the RNG performance. In this work, we are instead proposing a RNG design based on relative distribution of HRS. In this design, the main source of entropy is the cycle-tocycle HRS variation of a pair of memristors. With equal voltage bias of a fixed duration, the memristive states of two memristors are compared to generate random bit streams. Figure 1 displays the high level circuit diagram of the existing switching time based single memristor TRNG and the proposed relative HRS based twin memristor TRNG. Temperature, voltage change and process variation have smaller effect on this proposed TRNG performance as we'll analyze now.

Suppose we have two memristors which are physically close in a circuit layout and their mean HRS values are $1 \sigma=1 \%$ (mismatch) apart from each other where the variance of HRs is considered $10 \%$. It is justified to consider much smaller mismatch variation between any two devices if they are physically close in a chip compared to 
two devices very distant from each other in a chip or in different die. Each memristor's HRS has a cycle-to-cycle Normal distribution of mean $\mu_{H R S}$ and standard deviation $\sigma_{H R S}=10 \%$ of $\mu_{H R S}$.

$$
\begin{aligned}
& \mathrm{HRS}_{1}=N\left(\mu_{H R S 1}, \sigma_{H R S 1}\right) \\
& \mathrm{HRS}_{2}=N\left(\mu_{H R S 2}, \sigma_{H R S 2}\right)
\end{aligned}
$$

Since our proposed design compares between these HRS states, this can be expressed as another Normal random variable according to the property of Gaussian random variable.

$$
\begin{aligned}
\mathbf{Z} & =\mathrm{HRS}_{\mathbf{1}}-\mathrm{HRS}_{\mathbf{2}} \\
& =N\left(\left(\mu_{H R S 1} \sim \mu_{H R S 2}\right) \sqrt{\sigma_{H R S 1}^{2}+\sigma_{H R S 2}^{2}}\right)
\end{aligned}
$$

If $\mu_{H R S 2}>\mu_{H R S 1}$ and thus $\mu_{H R S 2}=1.01^{*} \mu_{H R S 1}$ (1\% larger) and $\sigma_{H R S 1}=0.1^{*} \mu_{H R S 1}, \sigma_{H R S 2}=0.1^{*} \mu_{H R S 2}(10 \%$ standard deviation $)$, then equation 13 becomes:

$$
\begin{aligned}
\mathrm{Z} & =N\left(.01 * \mu_{H R S 1}, \sqrt{\left(0.1 * \mu_{H R S 1}\right)^{2}+\left(0.1 * 1.01 \mu_{H R S 1}\right)^{2}}\right) \\
& \approx N\left(.01 * \mu_{H R S 1}, 0.1 * \sqrt{2} * \mu_{H R S 1}\right)
\end{aligned}
$$

Since $\mathbf{Z}$ doesn't have a mean of zero, the ratio of ones and zeros wouldn't be the same. However, it should be close to zero as can be deduced using a Z-table. The $\mathrm{z}$-score of this normal distribution to find the probability of a randomly chosen value to be less than 0 is:

$$
\begin{aligned}
z=\frac{x-\mu}{\sigma} & =\frac{0-0.01 * \mu_{H R S 1}}{0.1 * \sqrt{2} * \mu_{H R S 1}} \\
& =-0.0707
\end{aligned}
$$

where, $\mathrm{z}$ represents a Normal random variable of mean 0 and standard deviation 1, transformed from $\mathbf{Z}$ to calculate the probability using a z-table.

From z-table, the probability of getting a ' 0 ' value for z-score of -0.0707 is:

$$
\mathrm{P}(x \in \mathrm{Z} \leq 0)=0.4718
$$

It should be noted that if the mean HRS between two memristors are less than $1 \sigma$, then the the probability number in equation 16 would be even closer to the ideal value of 0.5 . Thus our proposed design inherently has some bias. But this provides us with a better robustness against environmental changes compared to existing designs as we'll analyze now.

\subsection{Supply voltage variation}

Changing the voltage applied across the memristors would change the switching speed. However, in our design, we chose a large enough switching time so that memristors would always switch, since we are only interested in variation in the final HRS values, not the switching probability itself. Therefore, due to rail voltage variation $(\approx \pm 10 \%)$, the time to generate a valid bit from the proposed TRNG changes slightly, but the value of that bit itself is assumed to be unaffected.

\subsection{Temperature variation}

Temperature would change the HRS of both memristors in our design. For a relatively small temperature window $[0-100]^{0} \mathrm{C}$, this change is approximated by a linear equation, similar to threshold voltage change. Suppose, the temperature increases by an amount of $\Delta \theta$. Then from equation 7 , we can find the two changed HRS as:

$$
\begin{aligned}
& \operatorname{HRS1}^{\prime}(\theta)=\operatorname{HRS} 1\left[1+\alpha_{H R S} * \Delta \theta\right] \\
& \operatorname{HRS}^{\prime}(\theta)=H R S 2\left[1+\alpha_{H R S} * \Delta \theta\right]
\end{aligned}
$$

where, $H R S_{1}$ and $H R S_{2}$ represent HRS at room temperature for these two different memristors. $\alpha_{H R S}$ is the linear temperature coefficient of HRS and approximated as $-0.008 /{ }^{\circ} \mathrm{C}$. The die-to-die standard deviation of HRS considered here is $\sigma_{H R S}=0.1^{*} \mu_{H R S}$ (10\% of HRS).

Now, let's use the term $C_{\Delta \theta}=1+\alpha_{H R S} * \Delta \theta$. Thus we can rewrite equations 12 for these new HRS values at a different temperature. The standard deviation (and variance) is considered unchanged.

$$
\begin{aligned}
\text { HRS }_{1}{ }_{1} & =N\left(\mu_{H R S 1^{\prime}}, \sigma_{H R S 1^{\prime}}\right) \\
& =N\left(\mu_{H R S 1} * C_{\Delta \theta}, \sigma_{H R S 1}\right) \\
\text { HRS }_{2}{ }_{2} & =N\left(\mu_{H R S 2^{\prime}}, \sigma_{H R S 2^{\prime}}\right) \\
& =N\left(\mu_{H R S 2} * C_{\Delta \theta}, \sigma_{H R S 2}\right)
\end{aligned}
$$

and

$$
\begin{aligned}
\mathbf{Z}^{\prime} & =\mathrm{HRS}_{1}^{\prime}-\mathrm{HRS}_{2}^{\prime} \\
& =N\left(C_{\Delta \theta} *\left(\mu_{H R S 1} \sim \mu_{H R S 2}\right), \sqrt{\sigma_{H R S 1}^{2}+\sigma_{H R S 2}^{2}}\right)
\end{aligned}
$$

Thus equation 18 and 19 actually represents scaled versions of the normal distribution of equation 12 and 13, respectively. Now we can find the new distribution of our proposed design by using equation 18 with equations 13 and 14 . The z-score of this scaled normal random variable is:

$$
\begin{aligned}
z=\frac{x-\mu}{\sigma} & =\frac{0-C_{\Delta \theta} * 0.01 * \mu_{H R S 1}}{0.1 * \sqrt{2} * \mu_{H R S 1}} \\
& =-C_{\Delta \theta} * 0.0707
\end{aligned}
$$

For $50^{\circ} \mathrm{C}$ temperature increase, $C_{\Delta \theta}=\left(1-.008^{*} 50\right)=0.6$ and thus the $\mathrm{z}$-score would be $\mathrm{z}=0.0424$. The probability of getting 0 :

$$
\mathrm{P}(x \in \mathrm{Z} \leq 0)=0.4831
$$

This value is very close to the value in equation 16 . Thus changing the temperature does not bring in a significant change on the ratio of 1's and 0's and the RNG performance does not degrade with changing temperature.

\subsection{Process variation}

In last subsection, we observe that temperature change creates a scaled version of the original HRS distributions. Process variation would cause the HRS values to be different from the die-to-die mean HRS value, causing a shift in the distribution. However, since we are taking the difference among two memristors, the resulting distribution would just display a scaled mean and scaled standard deviation of the original distribution.

$$
\begin{aligned}
\mathbf{Z} & =\mathrm{HRS}_{1}^{\prime \prime}-\mathrm{HRS}_{2}^{\prime \prime} \\
& =N\left(\left(\mu_{H R S 1^{\prime \prime}} \sim \mu_{H R S 2^{\prime \prime}}\right), \sqrt{\sigma_{H R S 1^{\prime \prime}}^{2}+\sigma_{H R S 2^{\prime \prime}}^{2}}\right) \\
& \approx N\left(.01 * \mu_{H R S 1^{\prime \prime}}, 0.1 * \sqrt{2} * \mu_{H R S 1^{\prime \prime}}\right)
\end{aligned}
$$


Table 1: Percentage of 0's to the sum of 0's and 1's for different operating conditions

\begin{tabular}{|c|c|c|c|c|c|c|c|}
\hline \multirow{2}{*}{$\begin{array}{c}\text { Design with } \\
\text { varying conditions }\end{array}$} & \multirow{2}{*}{$\begin{array}{c}\text { Ideal/No } \\
\text { change }\end{array}$} & \multicolumn{2}{|c|}{ Temperature variation } & \multicolumn{2}{|c|}{ Voltage variation } & \multicolumn{2}{|c|}{ Process variation } \\
\hline & & $+25^{0} \mathrm{C}$ & $-25^{0} \mathrm{C}$ & $+10 \%$ & $-10 \%$ & $+1 \sigma$ & $-1 \sigma$ \\
\hline Single-memristor & 50 & 4.01 & 95.99 & 18.16 & 86.67 & 69.15 & 30.85 \\
\hline Proposed (twin memristor) & 47.18 & 47.76 & 46.62 & 47.18 & 47.18 & 46.90 & 47.46 \\
\hline
\end{tabular}

Suppose, $\mu_{H R S 1^{\prime \prime}}$ is $1 \sigma$ larger than the mean $\mu_{H R S 1}$. Thus $\mu_{H R S 1^{\prime \prime}}$ $=1.1^{*} \mu_{H R S 1}$ and the $\mathrm{z}$-score of this would again be:

$$
\begin{aligned}
z=\frac{x-\mu}{\sigma} & =\frac{0-0.01 * \mu_{H R S 1^{\prime \prime}}}{0.1 * \sqrt{2} * \mu_{H R S 1}} \\
& =\frac{0-0.01 * 1.1 * \mu_{H R S 1}}{0.1 * \sqrt{2} * \mu_{H R S 1}} \\
& =-0.0778
\end{aligned}
$$

The probability of getting 0 for $1 \sigma$ variation of HRS is thus:

$$
\mathrm{P}(x \in \mathrm{Z} \leq 0)=0.469
$$

Thus the ratio of 1 's and 0 's change by a very small amount with process variation. This is an excellent result since it states that we do not need to change any design parameters even if memristors show a large variance across different die as the RNG performance should remain very close to optimum operating condition.

\subsection{Output Correction}

Since our proposed design inherently has a small percentage of bias i.e. amount of 1's and 0's produced are not equal even in ideal condition, some form of post processing could be useful to improve the data quality. In this design, we have proposed to use simple VonNeumann correction which can be implemented very easily with minimum hardware overhead. Von-Neumann correction discards when two consecutive RNG output bits are both ' 1 ' or ' 0 '. But if the bit sequence is ' 10 ' then it becomes a ' 1 ', and if the sequence is ' 01 ' then it becomes a ' 0 '. It helps to remove simple bias and reduce correlation between consecutive bits. By employing some 'whitening' technique, the the quality of the output bit can be improved too. XORing of two TRNGs is one such technique. If output from two TRNGs are not correlated, then XORing them could help to improve randomness in the output. XORing the TRNG output with the output from a PRNG could also improve randomness.

\section{RESULTS AND ANALYSES}

First, we have analyzed both the conventional single memristor based TRNG design and proposed twin memristor based TRNG design in terms of the proportion of 1's and 0's with respect to varying operating conditions. We have used a MATLAB version of Mcdonald model, developed in [14] for simulation. We have calculated the percentage of 0 's compared to the sum of 1 's and 0 's in normal operating condition, at temperature $\pm 25^{\circ} \mathrm{C}$ change compared to room temperature, applied voltage with $\pm 10 \%$ variation and with $1 \sigma$ process variation. This $1 \sigma$ process variation for single memristor design corresponds to the negative switching time variation and is assumed to be $5 \%$. For the proposed design, the variation corresponds to of HRS and is assumed to be $10 \%$ as HRS displays higher variation than switching time or other memristor characteristics. Table 1 displays the result. As expected, process variation
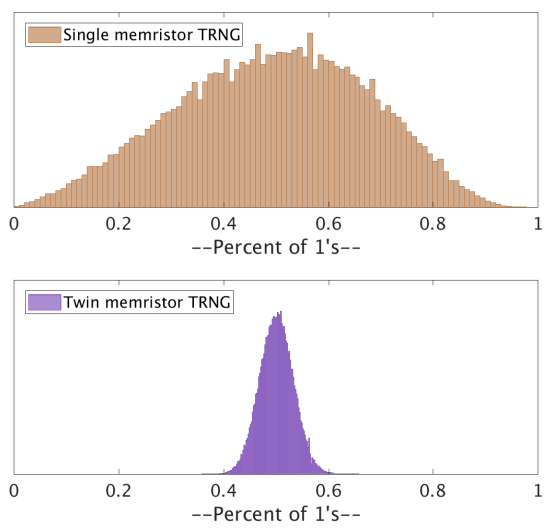

Figure 2: Histogram of bit bias with process variation for (top) existing single memristor design and (bottom) proposed twin memristor design

and temperature variation strongly affect switching time and thus the single memristor based design shows a large bias towards 1's or 0 's except for normal condition. The proposed design is based on relative measurement of HRS and because of mismatch, inherently has a small bias i.e. the proportion of 0 is not $50 \%$ ideally. However, due to its relative nature, our proposed design only show a small change in performance in varying operating conditions. Thus our design can be considered robust against environmental changes or against large process variation. Table 1 lists the performance of this two designs for different conditions. As expected, performance of the proposed design doesn't show a large deviation from ideal condition where a single memristor TRNG performance could be affected severely.

To better understand the effect of process variation, we have run Monte Carlo analysis with 10,000 different chips and generated 5000 random bits from each chip. Figure 2 shows the percentage of 1 's for different chips for both existing and proposed TRNG design. A TRNG should ideally have $50 \%$ of 1 's i.e. the mean should be 0.5 . As expected for our theoretical analysis, existing switching time based TRNG design shows a wide distribution with process variation with a standard deviation of 0.1805 . But our proposed twin memristor design only has a narrow distribution over die-to-die process variation with a standard deviation of only 0.0322 . This result shows our proposed design is robust against process variation and eliminates the need to redesign for every circuit instance.

We have also run Monte Carlo to visually show how the histogram shifts from ideal for a $\pm 10^{0} C$ change in temperature and for a $\pm 10 \%$ change in supply voltage. This result is shown in Figure 3. Conventional single memristor based design shows a large deviation from ideal (i.e. with a mean of 0.5) distribution for both 


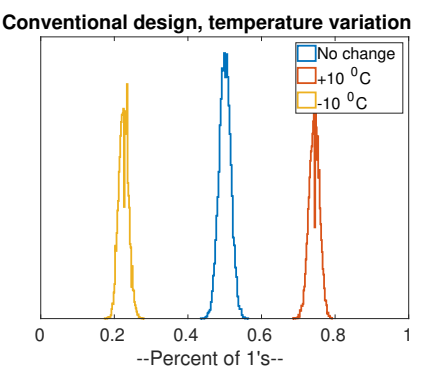

Conventional design, voltage variation
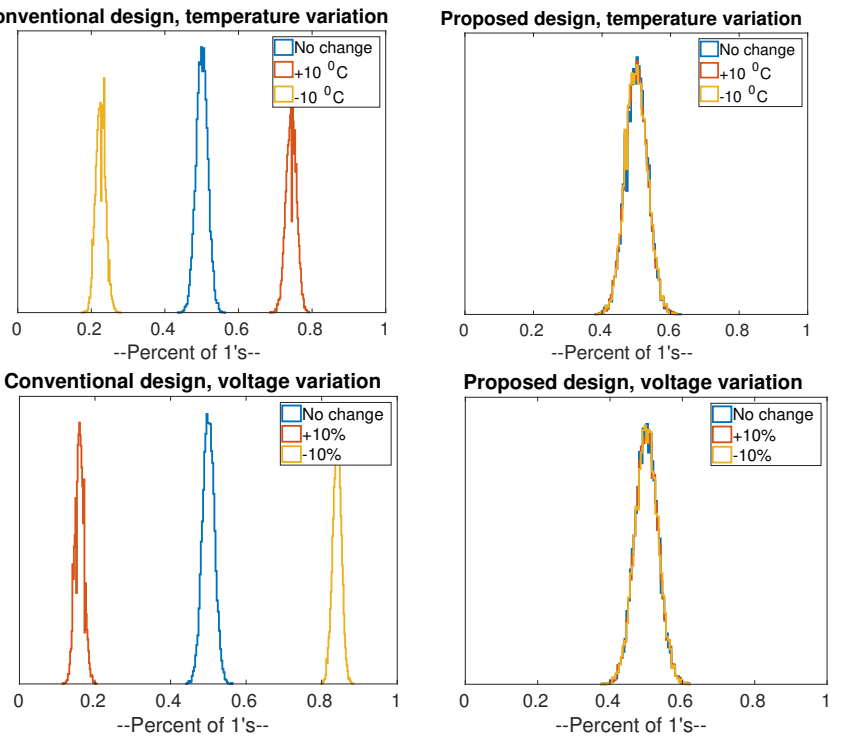

Figure 3: Histogram plot showing the shift in distribution with temperature and voltage variation for both conventional and proposed design. Conventional design displays a wide variation while proposed design almost unchanged.

increasing and decreasing temperature and supply voltage. But our proposed design shows almost zero or very negligible change.

\section{FUTURE WORK}

In this work, we have provided with a general idea about what to consider when designing a TRNG using memristor's stochastic switching behavior. However, different memristor materials would have different relationships with temperature and voltage as well as different variance of their parameters, one needs to characterize them first with corresponding temperature coefficients and switching equation to build a TRNG. Finally, our design is based on theoretical analysis only and thus other source of randomness (like noise) of a circuit might also affect the performance. Therefore, our immediate step would be to build our design using actual memristors and then evaluate it. The design of a good comparator to compare the memristive states is another important task. Finally, since our results are based on simplified mathematical model based simulation, any statistical random number tests are not shown in this work as software can only produce pseudo-random numbers.

\section{CONCLUSION}

We have used statistical distribution of random numbers to analyze the theoretical strength of existing TRNG designs against process, temperature and supply voltage variation. We have proposed our twin memristor based TRNG design which theoretically performs better than existing designs in these varying conditions. It also doesn't require sophisticated pulse width control to generate random bits and thus complexity of input signal generation circuit is reduced. However, as we know memristor is a novel device and its fabrication technology is still not mature, many different memristors consisting of many different materials with different underlying physics are out there. Thus the actual performance of a particular TRNG design would be very dependent on its underlying materials and their characteristics.

\section{ACKNOWLEDGMENT}

The authors would like to thank Md. Badruddoja Majumder and other members of our SENECA research group at the University of Tennessee for interesting discussions related to this topic.

This material is based upon work supported by the Air Force Office of Scientific Research under award number FA9550-16-10301. Any opinions, finding, and conclusions or recommendations expressed in this material are those of the authors and do not necessarily reflect the views of the United States Air Force.

\section{REFERENCES}

[1] Simone Balatti, Stefano Ambrogio, Zhongqiang Wang, and Daniele Ielmini. 2015. True random number generation by variability of resistive switching in oxidebased devices. IEEE Journal on Emerging and Selected Topics in Circuits and Systems (FETCAS) 5, 2 (2015), 214-221.

[2] T. Cabout et al. 2013. Temperature impact (up to 200C) on performance and reliability of $\mathrm{HfO}_{2}$-based RRAMs. In 5th IEEE International Memory Workshop. 116-119. https://doi.org/10.1109/IMW.2013.6582112

[3] Z. Fang, H. Y. Yu, W. J. Liu, Z. R. Wang, X. A. Tran, B. Gao, and J. F. Kang. 2010. Temperature Instability of Resistive Switching on $\mathrm{HfO}_{2}$-Based RRAM Devices. IEEE Electron Device Letter 31 (May 2010), 476-478. Issue 5.

[4] Hao Jiang, Daniel Belkin, Sergey E Savelév, Siyan Lin, Zhongrui Wang, Yunning $\mathrm{Li}$, Saumil Joshi, Rivu Midya, Can Li, Mingyi Rao, et al. 2017. A novel true random number generator based on a stochastic diffusive memristor. Nature communications 8,1 (2017), 882.

[5] Benjamin Jun and Paul Kocher. 1999. THE INTEL@ RANDOM NUMBER GENERATOR. Technical Report. CRYPTOGRAPHY RESEARCH, INC. WHITE PAPER PREPARED FOR INTEL CORPORATION. 8 pages.

[6] J. Kim, T. Ahmed, H. Nili, N. Duy Truong, J. Yang, D. S. Jeong, S. Sriram, D. C. Ranasinghe, and O. Kavehei. 2017. Nano-Intrinsic True Random Number Generation. ArXiv e-prints (Jan. 2017). arXiv: 1701.06020

[7] M. B. Majumder, M. Uddin, G. S. Rose, and J. Rajendran. 2016. Sneak path enabled authentication for memristive crossbar memories. In 2016 IEEE Asian Hardware-Oriented Security and Trust (AsianHOST). 1-6. https://doi.org/10.1109/ AsianHOST.2016.7835568

[8] Nathan R. McDonald. 2012. Al/Cu $u_{x} \mathrm{O} / \mathrm{Cu}$ Memristive Devices: Fabrication, Characterization, and Modeling. Master's thesis. College of Nanoscale Science and Engineering, University at Albany, Albany, NY.

[9] Rawan Naous, Maruan Al-Shedivat, and Khaled Nabil Salama. 2016. Stochasticity modeling in memristors. IEEE Transactions on Nanotechnology 15, 1 (2016), 15-28.

[10] P. Pouyan, E. Amat, and A. Rubio. 2014. Reliability challenges in design of memristive memories. In 5th European Workshop on CMOS Variability (VARI). 1-6. https://doi.org/10.1109/VARI.2014.6957074

[11] Dmitri B. Strukov, Gregory S. Snider, Duncan R. Stewart, and R. Stanley Williams. 2008. The missing memristor found. Nature 453 (May 2008), 80-83.

[12] M. Uddin, M.B. Majumder, H. Manem, K. Beckmann, Z. Alamgir, N. Cady, and G.S. Rose. 2017. Design Considerations for Memristive Crossbar Physical Unclonable Functions. Journal of Emerging Technology in Computing System (JETC) 14, 1, Article 2 (sep 2017), 23 pages. https://doi.org/10.1145/3094414

[13] M. Uddin, M.B. Majumder, G.S. Rose, H. Manem, K. Beckmann, Z. Alamgir, and N. Cady. 2016. Techniques for Improved Reliability in Memristive Crossbar PUF Circuits. In IEEE Computer Society Annual Symposium on VLSI (ISVLSI). 212-217.

[14] M. Uddin, M. B. Majumder, and G. S. Rose. 2017. Robustness Analysis of a Memristive Crossbar PUF Against Modeling Attacks. IEEE Transactions on Nanotechnology 16, 3 (May 2017), 396-405. https://doi.org/10.1109/TNANO.2017.2677882

[15] Christian Walczyk et al. 2011. Impact of Temperature on the Resistive Switching Behavior of Embedded $\mathrm{HfO}_{2}$-Based RRAM Devices. IEEE Transaction on Electron Devices 58 (September 2011), 3124-3131. Issue 9. https://doi.org/10.1109/TED. 2011.2160265

[16] Chaofei Yang, Beiye Liu, Yandan Wang, Yiran Chen, Hai Li, Xian Zhang, and Guangyu Sun. 2016. The applications of NVM technology in hardware security. In Proceedings of the 26th edition on Great Lakes Symposium on VLSI (GLSVLSI). ACM, 311-316.

[17] Cong Ye, Jiaji Wu, Gang He, Jieqiong Zhang, Tengfei Deng, Pin He, and Hao Wang. 2016. Physical mechanism and performance factors of metal oxide based resistive switching memory: a review. Journal of Materials Science \& Technology 32,1 (2016), 1-11. 